\title{
A demographic perspective on human wellbeing: Concepts, measurement and population heterogeneity
}

\author{
Sonja Spitzer $^{1, \dagger, *}$, Vanessa di Lego ${ }^{2, \dagger}$, Angela Greulich ${ }^{3}$ and \\ Raya Muttarak ${ }^{4}$
}

\section{Abstract}

This introduction to the 2021 special issue of the Vienna Yearbook of Population Research explores demographic perspectives on human wellbeing across time and space. While the idea of relating demographic parameters to wellbeing has been around for a while, a more concrete research agenda on this topic has only recently gained momentum. Reviewing the research presented in this volume, we show how existing theoretical concepts and methodological tools in demography can be used to make substantial advances in the study of wellbeing. We also touch upon the many challenges researchers face in defining and measuring wellbeing, with the most important debate being about whether the focus should be on objective or subjective measures. The studies discussed here define wellbeing as health and mortality; as income, education or other resources; as happiness or life satisfaction; or as a combination thereof. They cover wellbeing in historical and contemporary populations in high- and low-income countries, and also point out important barriers to research on wellbeing, including the lack of good quality data in many regions. Finally, we highlight the value of considering population heterogeneities when

\footnotetext{
${ }^{1}$ Department of Demography, University of Vienna, Wittgenstein Centre for Demography and Global Human Capital (IIASA, OeAW, University of Vienna), Vienna, Austria

${ }^{2}$ Vienna Institute of Demography (OeAW), Wittgenstein Centre for Demography and Global Human Capital (IIASA, OeAW, University of Vienna), Vienna, Austria

${ }^{3}$ Sciences Po - OSC and Institut Universitaire de France, Paris, France

${ }^{4}$ Population and Just Societies Program, International Institute for Applied Systems Analysis (IIASA), Wittgenstein Centre for Demography and Global Human Capital (IIASA, OeAW, University of Vienna), Laxenburg, Austria

${ }^{\dagger}$ These authors contributed equally to this work.

*Correspondence to: Sonja Spitzer, sonja.spitzer@univie.ac.at
} 
studying wellbeing in order to identify population subgroups who are likely to fall behind, which can have important policy implications.

Keywords: wellbeing; demography; subjective and objective measures; population heterogeneities

\section{Background}

Research on human wellbeing is often driven by the objective of "the greatest good for the greatest number" of people, a principle attributed to Jeremy Bentham, which is motivated by the desire to maximise wellbeing in a given population. However, since ancient Greece, there has been a longstanding debate about what constitutes wellbeing, and how it should be measured. While the hedonic Epicurean position was that wellbeing represents a life full of happiness, Aristotle proposed a definition of eudemonia, or of a life full of purpose, meaning and value. To date, it is still challenging to define and measure wellbeing, with the most important debates being about whether the focus should be on objective or subjective definitions of wellbeing, and about whether an individual or an aggregate perspective is the most useful for assessing wellbeing (Dodge et al. 2012; Voukelatou et al. 2020). Moreover, the approaches to conceptualising and measuring wellbeing differ substantially across disciplines. Economists, with their relatively long tradition of wellbeing research, have generally concentrated on objectively measurable dimensions, like GDP or household income (Reinhart et al. 2010). In contrast, sociologists and psychologists have tended to focus on a subjective concept of wellbeing that is linked to people's emotional and cognitive evaluations of their lives, psychosocial needs and an overall sense of purpose and emotional fulfilment (Diener et al. 2003; Tay and Diener 2011; Pleeging et al. 2021). These more subjective wellbeing indicators - like happiness and life satisfaction - have also recently been considered by other disciplines in the social sciences (Clark 2018).

As the scientific study of human population, demography seeks to measure various population characteristics, and to assess their drivers and implications. The ability to account for both macro- and micro-level changes across time and space allows for a broad, empirically based understanding of population trends and patterns (Billari 2015). This unique feature of demography makes the discipline ideal for the study of human wellbeing not only in the past and present, but also in the future, via forecasting (Muttarak et al. 2016). While the classic demographic events - birth, death and migration - have important links to wellbeing, mortality and health are used most frequently to conceptualise wellbeing.

In addition to existing theoretical and empirical tools, the ability to measure and incorporate population heterogeneity - i.e., variations among individuals in demographic behaviour, events and other outcomes - provides fruitful angles for the demographic analysis of wellbeing. Wellbeing differentials can be identified not only across the most commonly investigated population subgroups, like those based 
on age and gender, but also according to other key demographic characteristics, like level of education, relationship status and religion. Likewise, demography provides the resources for connecting these dimensions and analysing their interdependencies. By explicitly considering population heterogeneity, we increase our chances of preventing the infamous Benthamian pitfall of sacrificing the wellbeing of the small number for that of the greater number when seeking to maximise wellbeing.

This special issue of the Vienna Yearbook of Population Research serves as a starting point for exploring the contributions of demographic perspectives on the study of human wellbeing, while also bringing in different approaches and evidence from other scientific disciplines. It provides a deep dive into the concepts and measurements of human wellbeing in four contributions: two from the Demographic Debate; and two from Review Articles, in which the benefits and drawbacks of various wellbeing measures are discussed, along with their consequences for policymaking. Two additional Demographic Debates focus on specific policy targets, and on what is at stake when considering wellbeing. A further 13 Research Articles and a Data \& Trends contribution present novel empirical results on different wellbeing dimensions and indicators for various regions of the world. Thus, these contributions confirm demography's role as a highly relevant discipline for advancing the field of wellbeing.

\section{What is wellbeing in the demographic context, and how can we best measure it?}

What constitutes wellbeing is both a philosophical and practical question. As demography is open to a range of fields and disciplines, it has the advantage of being able to accommodate a myriad of understandings, concepts and ways of measuring wellbeing that derive from other areas. This openness is reflected in the contributions in this special issue, which attempt to capture the multidimensionality of wellbeing using a wide range of data and methods. Here, wellbeing is variously defined based on health or mortality; income, education or other resources; happiness or life satisfaction; or a combination thereof, usually in the form of a composite wellbeing indicator representing both objective and subjective dimensions of wellbeing.

Even within these various interpretations of wellbeing, there is still considerable room for discussion about how best to measure it - or, even more importantly, about who should measure it. When analysing human wellbeing, who should judge wellbeing levels: the individuals themselves, or an objective outsider based on a common set of criteria? In his Demographic Debate, Clark reviews the current discussion surrounding the most appropriate measurement of human wellbeing. $\mathrm{He}$ shows how objective wellbeing can be complemented with subjective measures, which have the advantage of being democratic; i.e., individuals decide what is important when assessing wellbeing. Information on subjective wellbeing can also 
be easily collected based on only one survey question, and it typically suffers less from missing values than variables needed for other operationalisations of wellbeing.

Simply asking individuals about their wellbeing has empirical benefits, and many of the contributions in this special issue have applied this subjective approach. Yildiz, Arslan and Cavlin, for example, study the determinants of subjective wellbeing in Turkey based on the survey question: "All things considered, how satisfied are you with your life as a whole?" By conceptualising wellbeing as selfreported life satisfaction, the authors are able to provide a global evaluation of wellbeing that can account for all life domains over an extended period of time, as perceived by the individuals themselves. Similarly, Zannella and De Rose study Italian parents' subjective wellbeing during the time they spend with their children. In particular, the impact of multitasking on subjective wellbeing related to time spent on childcare is evaluated based on the survey question: "How pleasant was the moment?" Again, the researchers rely on self-reports rather than objective sources when measuring wellbeing.

Lucas discusses the benefits and drawbacks of self-reported wellbeing in his Review. He focuses in particular on a major concern related to subjective wellbeing: namely, the challenge of providing global assessments of wellbeing. Global selfreports of wellbeing require individuals to consider and condense various life domains over a long period of time. Thus, such self-reports involve the tasks of remembering and aggregating - and the inability to perform these tasks effectively can distort the measurement of subjective wellbeing. In his contribution, he compares global self-reports of subjective wellbeing with experimental measures in which individuals evaluate their momentary experiences many times over a limited period of time. He concludes that while both types of measures have the potential to further our understanding of wellbeing, neither of them is without flaws.

These contributions thus pave the way for more "objective" measures of wellbeing to be considered. But which one of these measures is the most relevant? Historically, wellbeing has often been measured in monetary terms, a metric that - seemingly - allows wellbeing to be compared and aggregated. In particular, economists have generally considered GDP per capita relevant for measuring wellbeing. In the past decade, however, there has been a constant call to move away from using economic production as an indicator of social progress, and towards the adoption of a more holistic understanding of human wellbeing (Stiglitz et al. 2010; OECD 2021). It has become more common to complement purely economic measures with measures of social and environmental dimensions of quality of life. These dimensions can be measured at the aggregate and/or the individual level, and include, for example, employment, household debt, air and water quality, freedom of movement, freedom of family formation, freedom of political participation and access to institutions providing education and health services. The demographic measures that are used most often in this context are mortality and health, which are discussed in more detail in the next subsection. 


\section{Health and mortality as wellbeing concepts}

While being alive is a precondition for enjoying any form of wellbeing, being in good health is an essential component of human welfare, and is an important factor in economic development that has both intrinsic and instrumental value (Bloom et al. 2019). The World Health Organization has defined health as a state of complete physical, mental and social wellbeing, and not just as the absence of illness (World Health Organization 2020). Thus, wellbeing is explicitly associated with being healthy. Based on this definition, promoting health and wellbeing at older ages was established as one of the three priority areas outlined in the 2002 Madrid International Plan of Action on Ageing, which aimed to ensure that population ageing is sustainable and equitable (World Health Organization 2020). Ensuring healthy lives and promoting wellbeing among people of all ages is also one of the 17 Sustainable Development Goals (United Nations Development Programme 2015). In addition, in 2008, the EU Innovation Partnership on Active and Healthy Ageing set a target of achieving an increase of two Healthy Life Years by 2020; and in 2018, the UK government set a target to "ensure people can enjoy at least 5 extra healthy, independent years of life by 2035, while narrowing the gap between the experience of the richest and poorest". Despite these efforts, Jagger argues in her Demographic Debate that the gap in Healthy Life Years between European Union member states has widened. Indeed, past trends in disability-free life expectancy in England and projections suggest that it will be difficult to achieve an increase of five healthy and independent years by 2035 .

Several other contributions in this special issue also use health status to capture human wellbeing. Barbuscia and Comolli, for example, explore gender inequalities in Switzerland and France. The authors analyse the accumulation of gender inequalities with age using different measures of physical and mental health, as well as relational wellbeing. Their results show that while gender inequalities are greater for many dimensions of wellbeing in Switzerland than in France, socioeconomic inequalities in health and wellbeing are more pronounced in France than in Switzerland. The researchers also report that while gender inequalities tend to accumulate with age, educational gaps do not seem to diverge over time. These findings suggest that gender inequalities tend to be based on structural disadvantages that grow and accumulate with life events and the ageing process.

Similarly, Nepomuceno, di Lego and Turra focus on gender inequalities by considering the intersection of wellbeing, health and gender-specific issues in Latin America and the Caribbean. Their work presents empirical evidence on the gender paradox in health and mortality, whereby women live longer than men, but with higher rates of disability. In particular, the authors address the challenges of measuring this paradox in a unique environment characterised by high levels of socio-economic inequality that interact with gender- and age-specific disparities in health and mortality. When coupled with high levels of widowhood and impoverishment among older women, improvements in life expectancy may increase the burden of health for women, which may, in turn, negatively affect 
women's wellbeing (Monteverde et al. 2009). While the authors note that a lack of data hampered their efforts to investigate as many countries in the region as possible, the evidence they present confirms the persistence of the gender paradox for countries in Latin America and the Caribbean.

Indeed, variations in the measurement of wellbeing are determined not only by differences in the theoretical concepts and methods used, but also by differences in the availability and quality of data. Gaining access to appropriate data is often a challenge, particularly when studying the wellbeing of historical populations, as proper data may not be available for a given time period; or when studying the wellbeing of low-income countries, as data quality is often poor in such contexts. Problems with data quality can make it difficult to monitor trends in wellbeing or to set targets for policy interventions. Flici and Hammouda explore this topic by extensively evaluating the quality of mortality data in Algeria, and pointing out important data issues that can lead to biased interpretations. They investigate questionable observations, such as sudden changes in the mortality age pattern, and excess female mortality at reproductive ages that unexpectedly appears and disappears in specific years. The authors find that while the population census of 1998, which coincided with the end of the civil war, had better data quality, reliable information on mortality and health in Algeria is still lacking. In particular, the authors show how controlling for data quality and understanding irregular patterns can enable researchers to identify and explain the data issues in a country context with limited data.

Likewise, Harris shows that in the absence of survey or census data, alternative data sources may be used to fill in the gaps in the data for certain historical populations. The author demonstrates how anthropometric data from sources such as military recruitment or prison records can be used to study wellbeing in historic contexts. In his Review Article, Harris explores and assesses population height as a historical measure of wellbeing, noting that population health can reflect the economic and environmental conditions at the time of measurement.

\section{Education as another aspect of wellbeing}

While health is widely used as a key dimension of wellbeing, in a similar function, education can also be considered as both a means to achieve and an ultimate end of human wellbeing. The articles by Paul and Rashmi as well as by Bittmann operationalise education in this manner. Using panel survey data for India and Germany, respectively, they are able to capture how the subjective and objective school performance of children affects their educational outcomes and other dimensions of wellbeing later in life.

Paul and Rashmi investigate for India how children's educational wellbeing influences their school dropout and grade repetition rates measured seven years later. Educational wellbeing is assessed using objective and subjective measures based on both theoretical concepts and empirical results from a principal component analysis. 
They find that both objective and subjective measures of educational wellbeing are key determinants of educational outcomes, as measured by school dropout and grade repetition rates, even after controlling for relevant household and geographic characteristics.

Bittmann examines the effects of teachers' subjective assessments of which secondary school academic track is appropriate for each child, the level of compliance with the teachers' recommendations, and the socio-economic background of the parents on the subjective and objective educational outcomes of children in Germany. The author shows that pupils who chose to continue their secondary education on the academic track even though their teacher recommended a different track tend to perform worse on competency tests, to have lower grades, and to report lower levels of satisfaction and reading enjoyment than pupils who were advised to follow the academic track. Thus, Bittmann's results suggest that the teachers' assessments predict rather well how the students will perform academically in secondary school. Ignoring such a recommendation - which has been called academic track mismatch - can result in individuals having poor educational outcomes later in life, both objectively and subjectively. These two articles clearly highlight the importance of considering both the subjective and the objective aspects of wellbeing measurement, as well as the multidimensionality of wellbeing.

\section{Population heterogeneity in wellbeing}

Another important question for the study of wellbeing is how wellbeing differs across population subgroups, and whether certain demographic and socio-economic characteristics provide protective effects against the deterioration of wellbeing. Being able to identify which subgroups are likely to fall behind in wellbeing indicators could facilitate the design of policy interventions aimed at closing the gaps between groups.

Matsuo and Matthijs, for example, address heterogeneities by age and cohort by studying subjective wellbeing trajectories across generations. They find a $\mathrm{U}$-shaped relationship between age and happiness that reflects lower levels of happiness in mid-life, followed by an increase in happiness at older ages. The authors also note, however, that the patterns are rather flat, and show considerable diversity after age 80 in many countries. In terms of cohort effects, the baby boomers are found to have lower happiness levels than pre-boomers and post-boomers, which may indicate that baby boomers have been negatively affected by their early and formative life conditions and experiences. For example, economic inequality rose from the late 1960s onwards, in part because a larger cohort size created greater competition for schools and jobs. The authors argue that the cohort-specific factors of subjective wellbeing is an interesting field of future research, particularly for studying the Millennials, who are facing new challenges in adulthood that were not experienced by the previous generations. 
D'Albis, Clark and Greulich also investigate the U-shaped relationship between life satisfaction and age, with a focus on heterogeneities between partnered and unpartnered individuals. First, the authors demonstrate that the U-shape generally holds in Europe, but is flatter for partnered than for non-partnered individuals. They also show that gender differences in life satisfaction by age - namely, that women's life satisfaction is higher at younger ages and is lower at older ages - disappear after controlling for partnership status. This pattern is, however, less clear in the panel data, which the authors analyse in a second step. Commenting on these mixed results, the authors emphasise that the results on life satisfaction by age strongly depend on the type of data analysed. They also point out that to study how key demographic, economic and psychological variables change over the lifecycle, reliable longitudinal datasets are needed for a larger set of countries.

Riederer, Fritsch and Seewann also explore the impact of relationship status on wellbeing by comparing the subjective wellbeing of single households in Vienna with that of other household types. Their contribution introduces another layer to this special issue: namely, that of various dimensions of subjective wellbeing. Their findings show that singles in Vienna are less satisfied with their family life than two-adult households, but also that singles are more satisfied with their financial situation, leisure time and housing than households with children.

\section{Putting it all together: multidimensional approaches to sustainable wellbeing}

Several contributions in this special issue are concerned with the multidimensional aspect of wellbeing, while emphasising that all of the metrics discussed above are potentially valid measures of wellbeing, and that various demographic aspects should be considered when using wellbeing as a basis for decision-making. Like Bentham's principle of "the greatest good for the greatest number", the measurement of wellbeing is usually not a means to an end, but is, rather, a tool for evaluating and judging decisions or actions at various levels. These wellbeing indicators often aim to "go beyond GDP" by replacing a simple monetary metric with a more holistic measure that encompasses different quality of life domains. Accordingly, several indicators designed to capture the multidimensional nature of wellbeing, including both its objective and the subjective components, have been developed.

The Research Article by Bloom, Fan, Kufenko, Ogbuoji, Prettner and Yamey emphasises that per capita GDP has significant limitations when used as a standalone tool for measuring wellbeing, as it does not capture many dimensions associated with a "good life," such as health and equality of opportunity. Nonetheless, they acknowledge that GDP per capita has a number of advantages, including being easy to interpret and requiring data that are widely available for a large number of countries. With this motivation in mind, the authors propose a new parsimonious measure of wellbeing that preserves the advantages of per capita GDP, but that 
also includes health and equality. Thus, they offer an indicator that simultaneously accounts for income, health and inequality, and that can be readily interpreted as inequality-adjusted healthy lifetime income. This approach is useful for monitoring trends in wellbeing across countries and over time, and as an alternative strategy for performing cross-country comparative analysis.

Along these lines, Frijters critically discusses in his Demographic Debate article the practise of relying on cost-benefit analyses as the basis for making public spending decisions. As an alternative to assessing economic surplus, he suggests using the WELLBY indicator, which is one unit of self-reported life satisfaction on a scale of zero to 10 for one person for one year. To account for potential jealousy and envy effects - i.e., for the possibility that the higher consumption of others reduces an individual's wellbeing - the author proposes a reduction factor of $75 \%$ on all estimates of private consumption benefits for this new wellbeing framework. He suggests that decision-making should be based on the expected stream of WELLBYs, as estimated using the results of the several thousand studies on the determinants of life satisfaction.

The indicators mentioned above implicitly or explicitly take a demographic stance when measuring wellbeing. Frijters, for example, sums wellbeing over the population of interest. Similarly, Bloom, Fan, Kufenko, Ogbuoji, Prettner and Yamey include healthy life expectancy in their wellbeing indicator. In his Demographic Debate, Lutz emphasises that existing demographic tool kits, such as life tables and Sullivan methods, can be used to analyse human wellbeing, by, for example, calculating the person-years lived at any given age in the status of interest. This application of demographic methods allows for a straightforward and simple interpretation of wellbeing, as exemplified in Lutz, who introduces a new indicator of wellbeing called Years of Good Life (YoGL). Three of the empirical contributions in this special issue are dedicated to this newly introduced measure, which considers both subjective and objective measures of wellbeing, including selfreported life satisfaction, poverty and health. These research articles analyse YoGL across countries and population groups, as well as over time. Specifically, Striessnig, Reiter and Dimitrova reconstruct the indicator back to 1950 for 140 countries; Reiter and Spitzer use YoGL to decompose wellbeing by country and gender for the European population aged 50+; and Dimitrova and Buathong illustrate how the indicator can be applied using novel primary data collected in Thailand.

In his contribution, Lutz also outlines how demographic models, such as multidimensional demographic dynamics, can be used to project future population trends based on alternative scenarios. In particular, he notes that because demography is able to produce longer-term quantitative models, it can play a central role in efforts to account for the temporal dimension of changes in wellbeing. Such models can, in turn, be used to explore sustainable human wellbeing, which is the ultimate goal of sustainable development, while accounting for future challenges like climate change. Thus, holistic measures of human wellbeing are highly relevant for the Sustainable Development Agenda 2030, which seeks to achieve sustainable improvements in human wellbeing, while maintaining a balance between the environment and 
economic progress (Messerli et al. 2019). Monitoring the progress of sustainable development therefore requires overarching empirical measurements of human wellbeing.

Developing and constantly improving these wellbeing measures, as well as providing relevant data, particularly in low- and middle-income countries, are needed to formulate policies that promote wellbeing at the individual and the aggregate level. With contributions from authors from various countries, such as Algeria, India, Brazil, Turkey and Thailand, this special issue represents a first step towards producing evidence on wellbeing for countries outside of Europe and North America. This special issue also demonstrates that the study of wellbeing should consider population heterogeneity whenever possible, because failing to do so hampers efforts to identify population subgroups who are likely to fall behind in the development process. To promote policies designed to enhance wellbeing for the greatest number of people, a multidimensional approach that captures both subjective and objective dimensions, as well as different life domains, is needed, as the contributions in this volume vividly illustrate.

\section{References}

Billari, F. C. 2015. Integrating macro- and micro-level approaches in the explanation of population change. Population Studies 69: S11-S20. https://doi.org/10.1080/00324728. 2015.1009712

Bloom, D. E., M. Kuhn and K. Prettner 2019. Health and economic growth. Oxford Research Encyclopedia of Economics and Finance. https://doi.org/10.1093/acrefore/ 9780190625979.013.36

Clark, A. E. 2018. Four decades of the economics of happiness: Where next? Review of Income and Wealth 64(2): 245-269. https://doi.org/10.1111/ROIW.12369

Diener, E., S. Oishi and R. E. Lucas 2003. Personality, culture, and subjective well-being: Emotional and cognitive evaluations of life. Annual Review of Psychology 54(1): 403-425. https://doi.org/10.1146/annurev.psych.54.101601.145056

Dodge, R., A. Daly, J. Huyton and L. Sanders 2012. The challenge of defining wellbeing. International Journal of Wellbeing 2(3): 222-235. https://doi.org/10.5502/ijw.v2i3.4

Messerli, P., E. M. Kim, W. Lutz, J.-P. Moatti, K. Richardson, M. Saidam, D. Smith, P. Eloundou-Enyegue, E. Foli, A. Glassman, G. H. Licona, E. Murniningtyas, J. K. Staniškis, J.-P. van Ypersele and E. Furman 2019. Expansion of sustainability science needed for the SDGs. Nature Sustainability 2(10): 892-894. https://doi.org/10.1038/s41893-019-0394-z

Monteverde, M., K. Noronha and A. Palloni 2009. Effect of early conditions on disability among the elderly in Latin America and the Caribbean. Population Studies 63(1): 21-35. https://doi.org/10.1080/00324720802621583

Muttarak, R., W. Lutz and L. Jiang 2016. What can demographers contribute to the study of vulnerability? Vienna Yearbook of Population Research 13: 1-13. https://doi.org/10.1553/ populationyearbook $2015 \mathrm{~s} 1$ 
OECD 2021. Better life initiative: Measuring well-being and progress. OECD Better Policies for Better Lives. https://www.oecd.org/statistics/better-life-initiative.htm, accessed on 21 June 2021.

Pleeging, E., M. Burger and J. van Exel 2021. The relations between hope and subjective well-being: A literature overview and empirical analysis. Applied Research in Quality of Life 16(3): 1019-1041. https://doi.org/10.1007/s11482-019-09802-4

Reinhart, C. M., V. R. Reinhart, C. Reinhart and V. Reinhart 2010. After the Fall. NBER Working Paper 16334. https://doi.org/10.3386/w16334

Stiglitz, J. E., A. K. Sen and J.-P. Fitoussi 2010. Report by the commission on the measurement of economic performance and social progress. Paris: Commission on the Measurement of Economic Performance and Social Progress. https://ec.europa.eu/ eurostat/documents/8131721/8131772/Stiglitz-Sen-Fitoussi-Commission-report.pdf, last accessed 13 March 2015.

Tay, L. and E. Diener 2011. Needs and subjective well-being around the world. Journal of Personality and Social Psychology 101(2): 354-365. https://doi.org/10.1037/a0023779

United Nations Development Programme 2015. Human Development Report 2015. New York, NY. http://hdr.undp.org/en/content/human-development-report-2015

Voukelatou, V., L. Gabrielli, I. Miliou, S. Cresci, R. Sharma, M. Tesconi and L. Pappalardo 2020. Measuring objective and subjective well-being: dimensions and data sources. International Journal of Data Science and Analytics 11(4): 279-309. https://doi.org/ 10.1007/S41060-020-00224-2

World Health Organization 2020. Basic Documents: Constitution of the World Health Organisation. https://apps.who.int/gb/bd/pdf_files/BD_49th-en.pdf

Open Access This article is published under the terms of the Creative Commons Attribution 4.0 International License (https://creativecommons.org/licenses/by/4.0/) that allows the sharing, use and adaptation in any medium, provided that the user gives appropriate credit, provides a link to the license, and indicates if changes were made. 